\title{
RECOGNIZING THE REAL LINE
}

BY

YURI GUREVICH AND W. CHARLES HOLLAND

\begin{abstract}
A certain elementary statement about the group of automorphisms of the real line $\mathbf{R}$ is sufficient to characterize $\mathbf{R}$ among homogeneous chains. A similar result holds for the chain of rational numbers.
\end{abstract}

Is it possible to characterize the real line with elementary statements? The answer depends, of course, on the language used. At one extreme, using only the elementary language of ordered sets, the real line is indistinguishable from any other totally ordered set which is dense in itself and has no endpoints. At the other extreme, the usual ways of defining the real line use set quantification. A somewhat different approach which is valuable in many areas of mathematics, in particular, geometry, is to classify structures by properties of their automorphism group. In this spirit we distinguish the real line among homogeneous chains by a statement in the elementary language of the group of automorphisms of the line. The rational and irrational chains have the same group of automorphisms as each other, but they are distinguished from all other homogeneous chains by a statement in the same elementary language.

The problem was originally raised at the 1978 ordered group conference in Boise, and many of the methods of this paper were presented there and appear in [4]. The authors are grateful for several stimulating discussions with Andrew Glass. A slightly different approach to this and similar problems is developed independently by Jambu-Giraudet in [5], where, again, many of the results of this paper appear. Neither [4] nor [5] contains the theorems announced above. The crucial difference of the present paper with [4] and [5] begins with Lemma 1 of this paper.

For any totally ordered set (chain) $\Omega$, the group $A(\Omega)$ of all order-preserving permutations of $\Omega$ becomes a lattice-ordered group when ordered pointwise. These groups are fundamental in the study of lattice-ordered groups, since every latticeordered group can be embedded in some $A(\Omega)$ [3]. The elementary language appropriate to the lattice-ordered group $A(\Omega)$ contains the usual logical symbols and also symbols for equality, order, the group and lattice operations, and variables denoting members of $A(\Omega)$ (but does not contain variables denoting members of $\Omega$ ).

This paper was motivated by our interest in lattice-ordered groups, and most of the results were expressed and proved in that language. When this work was almost finished, we became aware of [5] where the interest is mainly in $A(\Omega)$ as a group. The lattice-ordered group ( $l$-group) $A(\Omega)$ is interpretable in the group $A(\Omega)$ in a

Received by the editors August 15, 1979 and, in revised form, March 15, 1980.

AMS (MOS) subject classifications (1970). Primary 03C65, 03C60; Secondary 06F 15.

(c) 1981 American Mathematical Society 0002-9947/81/0000-0261/\$03.00 
certain sense (see the appendix). If $\varphi$ is the $l$-group statement about $A(\mathbf{R})$ which characterizes $\mathbf{R}$ (where $\mathbf{R}$ denotes the real line), and $\phi^{\prime}$ is the translation of $\phi$ into the language of groups, then $\phi^{\prime}$ characterizes $\mathbf{R}$ also (because $\mathbf{R}$ is isomorphic to its inversion). The rational chain $\mathbf{Q}$ can be treated similarly. Hence, in the rest of this paper we deal with $A(\Omega)$ as an $l$-group.

The analogous question-which sets $\Omega$ are characterized by the elementary properties of the full symmetric group $S(\Omega)$ ?-has been investigated by McKenzie [7], Pinus [8], and Shelah [9].

Since $A(\mathbf{R})$ and $A([0,1])$ are isomorphic as lattice-ordered groups, we see it is necessary to impose some homogeneity condition before we can expect an arbitrary chain $\Omega$ to be completely characterized by properties of $A(\Omega)$. Therefore, we will assume throughout that $A(\Omega)$ acts transitively on $\Omega$.

Also, we will need the following facts about $A(\Omega)$, which can be found in [1]. The support of a member $f \in A(\Omega)$ is the set of points of $\Omega$ which are moved by $f$. A convex congruence on $\Omega$ is an equivalence relation which is preserved by the permutations in $A(\Omega)$ and whose classes are convex subsets of $\Omega$. The convex congruences on $\Omega$ are totally ordered by inclusion [3, Lemma 7]. For any convex congruence class $\Lambda, A(\Lambda)$ is isomorphic to a subgroup of $A(\Omega)$, namely those members of $A(\Omega)$ which fix each point off $\Lambda$. In particular, $A(\Lambda)$ is transitive on $\Lambda$, and every congruence class with more than one point contains the support of a nonidentity member of $A(\Omega)$. For any given convex congruence, all its classes are isomorphic to each other, by the transitivity of $A(\Omega)$. There are two cases to consider regarding the local structure of $\Omega$. In the first case, suppose there is no smallest convex congruence whose classes contain more than one point. Then every interval of $\Omega$ with more than one point contains a convex congruence class with more than one point, and hence contains the support of a nonidentity member of $A(\Omega)$. In the second case, suppose that there is a smallest convex congruence whose classes contain more than one point. If $\Lambda$ is a class of this congruence, then $A(\Lambda)$ has no nontrivial convex congruences on $\Lambda$, and there are just two possibilities [ 3 , Lemmas 16 and 17, and Theorem 4]. The first of these is that $A(\Lambda)$ is 0 -2-transitive ( $\Lambda$ is doubly homogeneous); that is, for all $\alpha, \beta, \gamma, \delta \in \Lambda$, if $\alpha<\beta$ and $\gamma<\delta$ then there exists $g \in A(\Lambda)$ such that $\alpha g=\gamma$ and $\beta g=\delta$. It is an easy exercise to verify that when $A(\Lambda)$ is 0 -2-transitive, $A(\Lambda)$ is also 0 - $n$-transitive for every positive integer $n$, in the sense that for every pair of sequences of points of $\Lambda, \alpha_{1}<\alpha_{2}$ $<\cdots<\alpha_{n}$ and $\beta_{1}<\beta_{2}<\cdots<\beta_{n}$, there exists $g \in A(\Lambda)$ such that $\alpha_{i} g=\beta_{i}$, for all $i$. In this case, for any interval $I$ of $\Omega$ containing more than one point, we may choose points $\alpha<\beta<\gamma<\delta$ all in $I$ and all in the same class $\Lambda$ of the minimal convex congruence. There exists $g \in A(\Lambda)$ such that $\alpha g=\alpha, \beta g=\gamma$, and $\delta g=\delta$. By truncation off the interval $(\alpha, \delta)$, we may assume the support of $g$ is contained between $\alpha$ and $\delta$, and hence lies in $I$. Here again, every interval of $\Omega$ containing more than one point contains the support of a nonidentity member of $A(\Omega)$. The only remaining possibility is that $A(\Lambda)$ is an archimedean totally ordered group with the property that if $e \neq g \in A(\Lambda)$ and $\lambda \in \Lambda$, then $\left\{\lambda g^{n} \mid n=\right.$ $0, \pm 1, \pm 2, \ldots\}$ has no upper or lower bound in $\Lambda$. In this case, $\Omega$ is said to be 
locally Ohkuma and $\Lambda$ is an Ohkuma segment (see [1] or [3] for more details). To recapitulate: either every interval of $\Omega$ with more than one point contains the support of a nonidentity member of $A(\Omega)$, or $\Omega$ is locally Ohkuma.

In any case, the members of $A(\Omega)$ have unique extensions to $A(\bar{\Omega})$, where $\bar{\Omega}$ is the Dedekind completion of $\Omega$. A member $f \in A(\Omega)$ is positive if $f>e$, where $e$ is the identity permutation. For any $f \in A(\Omega),|f|=f \vee f^{-1}$. Two elements $f, g \in A(\Omega)$ are disjoint if $|f| \wedge|g|=e$, which is equivalent to saying that the supports of $f$ and $g$ are disjoint sets. A convex cycle is a member of $A(\Omega)$ whose support in $\bar{\Omega}$ consists of a single interval. Convex cycles $f$ are characterized by the property that if $\alpha f \neq \alpha$ then the set $\left\{\alpha f^{n} \mid n=0, \pm 1, \pm 2, \ldots\right\}$ is cofinal and coinitial in the support of $f$. Roughly speaking, the graph of a convex cycle has "one bump" in comparison with the identity function. It is instructive to note that a member of $A(\Omega)$ whose support consists of a single interval in $\Omega$ need not be a convex cycle, since its graph could have two bumps separated by a point of $\bar{\Omega}$ not in $\Omega$. Every positive member of $A(\Omega)$ is uniquely the join of a set of pairwise disjoint convex cycles.

Our general aim is to translate certain statements about $\Omega$ or $A(\Omega)$ into equivalent elementary statements about $A(\Omega)$. The first of these is $g$ is a positive convex cycle.

It is easily seen that this is equivalent to the elementary statement: $e<g$ and not ( $\exists f, h$ such that $g=f \vee h$ and $f \wedge h=e$ and $f \neq e$ and $h \neq e$ ).

$$
\begin{aligned}
& g \mathcal{L} h \quad \text { The support of } g \text { lies to the left of the support of } h \text {; that is, if } \\
& \alpha g \neq \alpha \text { and } \beta h \neq \beta \text {, then } \alpha<\beta \text {. }
\end{aligned}
$$

An equivalent statement is: $\forall f\left(f \geqslant e \Rightarrow|g| \wedge\left|f^{-1} h f\right|=e\right)$. The equivalence is clear when one observes that the support of $f^{-1} h f$ is the image under $f$ of the support of $h$.

$\mathscr{B}(g) \quad g$ has bounded support.

This is equivalent to: $\exists f, h(f \varrho g$ and $g \varrho h$ and $f \neq e$ and $h \neq e)$.

In order to translate statements about points of $\Omega$ or $\bar{\Omega}$ into statements about $A(\Omega)$, we want to identify each point $\alpha$ of $\bar{\Omega}$ with the set of those members of $A(\Omega)$ whose support has least upper bound $\alpha$. Two permutations corresponding to the same point in this way are similar in the sense of the following statement.

$g \sim h \quad$ The supports of $g$ and $h$ have the same least upper bound in $\bar{\Omega}$.

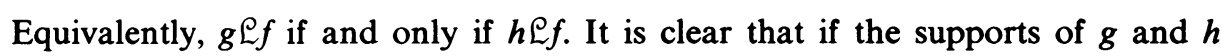
have the same least upper bound then the elementary statement holds. Conversely, if l.u.b. support $g=\alpha<\beta=1$.u.b. support $h$ and every interval of $\Omega$ supports a

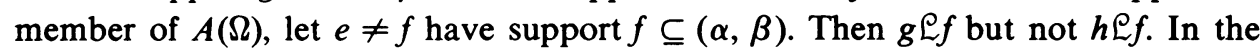
other case, if $\gamma \in(\alpha, \beta)$ and $I$ is the Ohkuma segment containing $\gamma$, then every point of $I$ is above every point of support $g$, but some point of $I$ is below some point of support $h$, so if $e \neq f$ and support $f \subseteq I$, then $g \varrho f$ but not $h \varrho f$.

A similar argument shows

$$
\operatorname{Adj}(g, h) \quad \text { l.u.b. support } g=\text { g.l.b. support } h \text { (in } \bar{\Omega})
$$

is equivalent to $g \varrho h$, and for no $f \neq e$ is $g \varrho f$ and $f \varrho h$. 
This is equivalent to $\exists f>e$ (for all $g, h, e \leqslant g<f$ and $e<h<f \Rightarrow g<h$ or $h \leqslant g$ ). If $A(\Omega)$ is locally Ohkuma, then any $f>e$ whose support lies in an Ohkuma segment works. Conversely, if $f$ has the property described in the statement, then the support of $f$ is an Ohkuma segment.

It is easy to see that $A(\Omega)$ is 0 -2-transitive if and only if for each $\alpha<\beta<\gamma$ in $\Omega$, there exists $f \in A(\Omega)$ such that $\alpha f=\alpha$ and $\gamma \leqslant \beta f$. From this, it is easily proved that

$$
A(\Omega) \text { is } 0 \text {-2-transitive }
$$

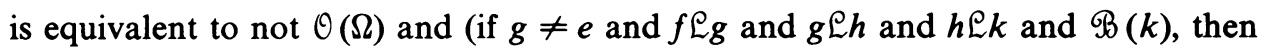
$\exists j$ such that $|f| \wedge j=e$ and $k \varrho^{-1} h j$ ).

Suppose $\mathcal{H}(\Omega)$. Then certainly not $\mathcal{\theta}(\Omega)$, and if $\mathscr{B}(k)$ and $f \mathscr{L} g \mathcal{L} h \mathcal{L} k$, choose $\alpha, \beta, \gamma \in \Omega$ with $\alpha<\beta, \alpha, \beta \in \operatorname{supp} g$, and $\operatorname{supp} k<\gamma$. Then there exists $j \in$ $A(\Omega)$ such that $e \leqslant j, \delta j=\delta$ for all $\delta \leqslant \alpha$, and $\beta j=\gamma$. It follows that $|f| \wedge j=e$ and $\operatorname{supp} k<\gamma=\beta j<(\operatorname{supp} h) j=\operatorname{supp}\left(j^{-1} h j\right)$, so $k \mathscr{L} j^{-1} h j$. Conversely, suppose the elementary statement is true, and let $\alpha<\beta<\gamma$ with $\alpha, \beta, \gamma \in \Omega$. Choose $\alpha_{i} \in \Omega$ so that $\alpha<\alpha_{1}<\alpha_{2}<\beta<\gamma<\alpha_{3}$. As $\Omega$ is not locally Ohkuma, every interval of $\Omega$ supports a member of $A(\Omega)$. Choose nontrivial $f, g, h, k \in A(\Omega)$ so that $\alpha<\operatorname{supp} f<\alpha_{1}<\operatorname{supp} g<\alpha_{2}<\operatorname{supp} h<\beta<\gamma<\operatorname{supp} k<\alpha_{3}$. Then

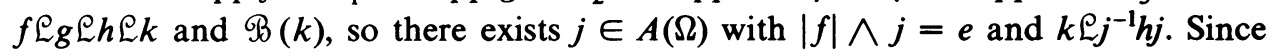
$\delta j=\delta$ for each $\delta \in \operatorname{supp} f$, we may assume that $\delta j=\delta$ for all $\delta<\operatorname{supp} f$, and in particular, $\alpha j=\alpha$. Moreover, $\beta j>(\operatorname{supp} h) j=\operatorname{supp}\left(j^{-1} h j\right)>\operatorname{supp} k>\gamma$. Hence $\mathcal{H}(\Omega)$.

The next statement has a decidedly nonelementary flavor.

$\Omega$ is Dedekind complete and not discrete.

This is equivalent to: $\mathcal{H}(\Omega)$ and (if $\mathscr{B}(g)$ and $g \neq e$ then $\exists h$ such that $\left.\operatorname{Adj}\left(g, h^{-1} g h\right)\right)$.

To prove this, assume $\Omega$ is Dedekind complete and not discrete. It is known that $A(\Omega)$ is 0 -2-transitive [3]. Furthermore, if $\mathscr{B}(g)$, let $\alpha=$ g.l.b. support $g$ and $\beta=$ l.u.b. support $g$. Then $\alpha, \beta \in \Omega$ and if $\alpha h=\beta$, it is obvious that $\operatorname{Adj}\left(g, h^{-1} g h\right)$. Conversely, if $\Omega$ is discrete there are points $\alpha<\beta<\gamma$ of $\Omega$ with no other points of $\Omega$ between $\alpha$ and $\gamma$. No order-preserving permutation of $\Omega$ can map $\alpha$ to $\alpha$ and $\gamma$ to $\beta$. Hence not $\mathcal{H}(\Omega)$. Now suppose, instead, that $\Omega$ is not Dedekind complete. Choose $\alpha<\beta, \alpha \in \Omega, \beta \in \bar{\Omega} \backslash \Omega$. Assuming $\mathcal{H}(\Omega)$, we easily construct $g \in A(\Omega)$ such that $\alpha=$ g.l.b. support $g$ and $\beta=1$.u.b. support $g$ as follows. Because $\mathcal{H}(\Omega)$, every nontrivial interval of $\Omega$ contains the support of some positive member of $A(\Omega)$, and so we can produce, by transfinite induction if necessary, a pairwise disjoint collection $\left\{g_{i}\right\}$ of positive members of $A(\Omega)$ in this manner: Let $e<g_{0}$ and support $g_{0} \subseteq(\alpha, \beta)$. If l.u.b. support $g_{0}=\beta_{0}<\beta$, then let $e<g_{1}$ and support $g_{1}$ $\subseteq\left(\beta_{0}, \beta\right)$. Continue in this manner until l.u.b. $\cup$ support $g_{i}=\beta$. In a similar way, we can arrange that g.l.b. $\cup$ support $g_{i}=\alpha$. Then $g=\bigvee g_{i}$ exists and has the desired property. Now if $h \in A(\Omega)$ and $g \varrho h^{-1} g h$, it must be that $\beta<\alpha h$. Hence,

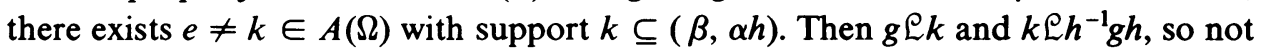
$\operatorname{Adj}\left(g, h^{-1} g h\right)$. 
This is clearly equivalent to: $C(g)$ and not $(\exists f \neq e$ such that $f \wedge g=e)$.

Lemma 1. Assume $\mathcal{H}(\Omega), G$ a subgroup of $A(\Omega)$, and for each $e \neq g \in G, \mathcal{G}(g)$ or $g\left(g^{-1}\right)$.

(a) Then the following are equivalent:

(1) For all $\alpha \in \bar{\Omega}, \alpha G$ is dense in $\bar{\Omega}$.

(2) If $e \neq f \in A(\Omega)$ and $f g=$ gf for all $g \in G$, then $\mathcal{G}(f)$ or $\mathcal{G}\left(f^{-1}\right)$.

(b) If $G$ satisfies (1) and (2) of part (a) and $H$ is a subgroup of $G$, then $H$ satisfies (1) and (2) of part (a) if and only if $\mathrm{H}$ is not cyclic.

COROllary. If $\mathcal{H}(\Omega)$, the following elementary statement implies that $\Omega$ has a countable order-dense subset:

There exist $f_{1}, f_{2}$ in $A(\Omega)$ such that $f_{1} f_{2}=f_{2} f_{1}$, and if $e \neq g \in A(\Omega)$ such that $g f_{i}=f_{i} g$, then $G(g)$ or $G\left(g^{-1}\right)$. Of course, the statement with any other finite number of $f_{i}$ also implies the same conclusion.

Proof of Lemma 1. (a) Assume (1) and $e \neq f, f g=g f$ for all $g \in G$. If $\alpha f=\alpha$ for some $\alpha \in \bar{\Omega}$, then $\alpha g f=\alpha g$ for all $g \in G$. Since $f$ fixes each point of the dense subset $\alpha G, f=e$, a contradiction. Hence, without loss of generality, $\alpha<\alpha f$ for all $\alpha \in \bar{\Omega}$, and $\mathscr{G}(f)$. Thus, (1) implies (2). Now assume there exists $\alpha \in \bar{\Omega}$ with $\alpha G$ not dense in $\bar{\Omega}$. Let $\Sigma$ be a maximal nontrivial interval of $\bar{\Omega}$ which contains no points of $\alpha G$. Because $\mathcal{H}(\Omega)$, there exists $e \neq \hat{f} \in A(\Omega)$ with support $\hat{f} \subseteq \Sigma$. Suppose that for some $g \in G, \Sigma \cap \Sigma g$ is not empty. It follows that $\Sigma=\Sigma g$, so neither $g(g)$ nor $9\left(g^{-1}\right)$; hence $g=e$. Consequently, for $g, h \in G$, if $\Sigma g \cap \Sigma h$ is not empty, then $g=h$. We may now unambiguously define $f \in A(\Omega)$ by

$$
f= \begin{cases}g^{-1} \hat{f} g & \text { on each } \Sigma g, g \in G, \\ e & \text { otherwise. }\end{cases}
$$

Then $f$ commutes with each member of $G$. But $f \neq e$, and yet $f$ fixes the endpoints of $\Sigma$ (in $\bar{\Omega}$ ), so not $g(f)$ and not $\mathscr{G}\left(f^{-1}\right)$. Hence (2) implies (1).

(b) By the assumption, for every $g \in G$, either $g>e$ or $g<e$, so $G$ is totally ordered. If $e<g<f$ and $g, f \in G$, then for any $\alpha \in \Omega,\left\{\alpha g^{n} \mid n=1,2,3, \ldots\right\}$ is cofinal in $\Omega$ (because $g(g)$ ), and hence, for some positive integer $n, \alpha f<\alpha g^{n}$. Therefore $g^{n} \nless f$, and since $G$ is totally ordered, $f<g^{n}$. This shows that $G$ is Archimedean. If $H$ is not cyclic, then $H$ is dense in $G$. In this case, if $\alpha, \beta, \gamma \in \Omega$ with $\beta<\gamma$, then there exist $f, g \in G$ such that $\beta<\alpha f<\alpha g<\gamma$, and there exists $h \in H$ such that $f<h<g$. It follows that $\beta<\alpha f<\alpha h<\alpha g<\gamma$. Hence $H$ has a dense orbit. Conversely, it is obvious that a cyclic group cannot have a dense orbit.

For the remainder of this paper, we let $\Omega$ denote a chain isomorphic either to the chain $\mathbf{R}$ of real numbers or the chain $\mathbf{Q}$ of rational numbers. In the former case, we may assume $\Omega=\mathbf{R}$; and in the latter case, we assume $\Omega$ is the additive subgroup of $\mathbf{R}$ generated by 1 and $\sqrt{2}$ (since any two chains, each countable and dense in itself without endpoints, are isomorphic). Let $t: x \mapsto x+1$ and $s: x \mapsto x+\sqrt{2}$, so $t, s \in A(\Omega)$. Then the members of the subgroup $G$ of $A(\Omega)$ generated by $t$ and $s$ are just the translations of the form $x \mapsto x+m+n \sqrt{2}$ with $m, n$ integers. Also, 
$\mathcal{H}(\Omega)$. Since every orbit of $G$ is dense, Lemma 1 implies that if $e \neq f \in A(\Omega)$ and $f g=g f$ for all $g \in G$, then $g(f)$ or $\mathscr{G}\left(f^{-1}\right)$.

Now suppose that $\Lambda$ is a chain such that $A(\Lambda)$ is transitive and $A(\Lambda)$ is elementarily equivalent to $A(\Omega)$. The $\mathcal{H}(\Lambda)$, and there exist $\bar{s}, \bar{t} \in A(\Lambda)$ such that $\bar{s} \bar{t}=\bar{t} \bar{s}$, and for each $e \neq f \in A(\Lambda)$ such that $\bar{s} f=f \bar{s}$ and $\bar{t} f=f \bar{t}, \mathscr{G}(f)$ or $\mathscr{G}\left(f^{-1}\right)$. By the corollary to Lemma $1, \Lambda$ has a countable subset which is dense in $\Lambda$. Consequently, $\Lambda$ is isomorphic to a dense subset of $\mathbf{R}$. In case $\Omega=\mathbf{R}$, then $\mathscr{D}(\Omega)$, so also $\mathscr{D}(\Lambda)$, which implies $\Lambda$ is isomorphic to $\mathbf{R}$. Thus we have our main result.

THEOREM. Let $\mathbf{R}$ be the chain of real numbers. There is a formula $\rho$ in the elementary language of groups such that $\rho$ holds in $A(\mathbf{R})$, and if $\Lambda$ is a homogeneous chain and $\rho$ holds in $A(\Lambda)$, then $\Lambda$ is isomorphic to $\mathbf{R}$.

We go on now to deal with the case when $\Omega$ is countable, and where there is a substantial extra difficulty. The lattice-ordered group $A(\mathbf{Q})$ is isomorphic to the lattice-ordered group $A(\mathbf{R} \backslash \mathbf{Q})$ (extension to $\mathbf{R}$ followed by restriction provides an isomorphism from either group to the other). Thus, it will certainly not be possible to distinguish $\mathbf{Q}$ from $\mathbf{R} \backslash \mathbf{Q}$ on the basis of elementary statements about $A(\mathbf{Q})$ and $A(\mathbf{R} \backslash \mathbf{Q})$. We will show, however, that this is the worst that can happen; that is, if $A(\Lambda)$ is elementarily equivalent to $A(\mathbf{Q})$, then $\Lambda \approx \mathbf{Q}$ or $\Lambda \approx \mathbf{R} \backslash \mathbf{Q}$.

Assume as before, that $\Omega$ is the additive subgroup of $\mathbf{R}$ generated by 1 and $\sqrt{2}$, and that $\langle t, s\rangle$ is the subgroup of $A(\Omega)$ generated by the translations $t$ and $s$. Let $\mathbf{Z}(t, s)=\{f \in A(\Omega) \mid f t=t f$ and $f s=s f\}$. Note that $\mathbf{Z}(t, s)=\langle t, s\rangle$, because certainly $\langle t, s\rangle \subseteq \mathbf{Z}(t, s)$ since $t s=s t$, and on the other hand, if $f \in \mathbf{Z}(t, s)$, then there exists $g \in\langle t, s\rangle$ such that $0 f=0 g$ (zero $=0 \in \mathbf{R}$ ). We show that $f=g$. For any $\alpha \in \Omega$, there exists $h \in\langle t, s\rangle$ such that $0 h=\alpha$. Then $\alpha f=0 h f=0 f h=0 g h$ $=0 h g=\alpha g$, so that $f=g$. Hence $Z(t, s) \subseteq\langle t, s\rangle$. For any subset $G$ of $A(\Omega)$, let $\mathcal{T}(G)$ stand for the statement: $G$ satisfies the condition $(a)(2)$ in Lemma 1 . Note that $\mathscr{T}(G)$ is equivalent to $\mathscr{T}(\langle G\rangle)$, where $\langle G\rangle$ denotes the subgroup generated by G.

The following are true:

(i) $t, s \in \mathbf{Z}(t, s)$ and $t>e$ and $s>e$;

(ii) $\mathcal{T}(\{t, s\})$;

(iii) if $f \in \mathbf{Z}(t, s)$ and not $\mathcal{T}(\{t, f\})$ then $t \leqslant|f|$;

(iv) if $f \in \mathbf{Z}(t, s)$ and not $\mathcal{T}(\{s, f\})$ then $s \leqslant|f|$;

(v) if $f \in \mathbf{Z}(t, s)$ then $\exists g, h$ such that $f=g h$ and $g, h \in \mathbf{Z}(t, s)$ and not $\mathcal{T}(\{t, g\})$ and not $\mathcal{T}(\{s, h\})$.

Parts (iii)-(v) are clear because, for example, $f \in \mathbf{Z}(t, s)$ and not $\mathcal{T}(\{t, f\})$ is equivalent to $f \in\langle t\rangle$.

Assuming that $A(\Lambda)$ is transitive and elementarily equivalent to $A(\Omega)$, since (i)-(v) are elementary statements we may assume there exist $\bar{t}, \bar{s} \in A(\Lambda)$ satisfying the appropriately relabelled forms of (i)-(v). Then the hypotheses of Lemma 1 are satisfied for $G=\mathbf{Z}(\bar{t}, \bar{s})$, and $\mathcal{T}(G)$. Thus, if $f \in G$, then $f=g h$ with $g, h \in G$ and not $\mathcal{T}(\langle\bar{t}, g\rangle)$ and not $\mathcal{T}(\langle\bar{s}, h\rangle)$. From Lemma $1(\mathrm{~b})$ it follows that $\langle\bar{t}, g\rangle$ and $\langle\bar{s}, h\rangle$ are cyclic. Let $q$ be the positive generator of $\langle\bar{t}, g\rangle$, so that $q<\bar{t}$. Then 
$q \in G$ and not $\mathcal{T}(\langle q\rangle$ ), so $\bar{t} \leqslant q$ (from (iii)). Hence $\bar{t}=q$, and $g \in\langle\bar{t}\rangle$. Likewise, $h \in\langle\bar{s}\rangle$. We conclude that $G=\langle\bar{t}, \bar{s}\rangle$, and therefore $\mathbf{Z}(\bar{t}, \bar{s})$ is countable. Finally, we are going to exploit the fact that $\langle t, s\rangle(=\mathbf{Z}(t, s))$ acts transitively on $\Omega$. Let $c \in A(\Omega)$ such that $\mathcal{C}(c), \mathscr{B}(c)$, and l.u.b. support $c=\alpha \in \Omega$. Then if $f \in A(\Omega)$, l.u.b. support $f^{-1} c f=\alpha f \in \Omega$, so there exists $g \in \mathbf{Z}(t, s)$ such that $\alpha g=\alpha f$, and hence $f^{-1} c f \sim g^{-1} c g$. In short: $\exists c$ such that $\mathcal{C}(c)$ and $\mathscr{B}(c)$ and $\forall f \exists g(g \in \mathbf{Z}(t, s)$ and $\left.f^{-1} c f \sim g^{-1} c g\right)$. The corresponding statement must be true for some $\bar{c} \in A(\Lambda)$, and there are two cases.

Case 1. l.u.b. support $\bar{c}=\beta \in \Lambda$.

Then if $\lambda \in \Lambda$, there exists $f \in A(\Lambda)$ such that $\beta f=\lambda$, and so since $f^{-1} \bar{c} f \sim g^{-1} \bar{c} g$ for some $g \in \mathbf{Z}(\bar{t}, \bar{s})$, we have $\beta g=\lambda$, and thus $\mathbf{Z}(\bar{t}, \bar{s})$ acts transitively on $\Lambda$. Consequently, $\Lambda$ is countable and therefore isomorphic to $\mathbf{Q}$.

Case 2. l.u.b. support $\bar{c}=\beta \in \mathbf{R} \backslash \Lambda$.

In this case, for every $\gamma \in \mathbf{R} \backslash \Lambda$ there exists $f \in A(\Lambda)$ such that $\beta f=\gamma$ (this is easy to prove using 0 -2-transitivity of $A(\Lambda)$ together with the fact that every point of $\mathbf{R}$ has countable character). Since $f^{-1} \bar{c} f \sim g^{-1} \bar{c} g$ for some $g \in \mathbf{Z}(\bar{t}, \bar{s})$, then $\beta g=\gamma$, and $\mathbf{Z}(\bar{t}, \bar{s})$ acts transitively on $\mathbf{R} \backslash \Lambda$. Thus $\mathbf{R} \backslash \Lambda$ is countable. Since $\mathcal{T}(\mathbf{Z}(\bar{t}, \bar{s}))$, the orbit $\mathbf{R} \backslash \Lambda$ is dense in $\bar{\Lambda}=\mathbf{R}$. It follows that $\Lambda$ is isomorphic to the chain $\mathbf{R} \backslash \mathbf{Q}$ of irrational numbers.

THEOREM. Let $\mathbf{Q}$ be the chain of rational numbers and $\mathbf{R}$ the chain of real numbers. There is a formula $\delta$ in the elementary language of groups such that $\delta$ holds in $A(\mathbf{Q})$ and in $A(\mathbf{R} \backslash \mathbf{Q})$, and if $\Lambda$ is a homogeneous chain and $\delta$ holds in $A(\Lambda)$, then $\Lambda$ is isomorphic to either $\mathbf{Q}$ or $\mathbf{R} \backslash \mathbf{Q}$.

Appendix.

Lemma 2 (MCCleary [6]). If $\Omega$ is doubly homogeneous, and $p \in A(\Omega)$, then ( $p>e$ or $p<e)$ if and only if there exist nontrivial $f, g \in A(\Omega)$ such that for all $u \in A(\Omega), f$ commutes with $\left(u^{-1} p u\right) g\left(u^{-1} p u\right)^{-1}$.

The following lemma is easily proved.

LEMMA 3 (SEE [5]). There is an elementary group formula $\phi_{p}(x, y)$ such that for every $p, x, y$ in the 0-2-transitive $A(\Omega)$,

(i) if $p>e$ the $\phi_{p}(x, y)$ holds in $A(\Omega)$ if and only if $x<y$,

(ii) if $p<e$ then $\phi_{p}(x, y)$ holds in $A(\Omega)$ if and only if $y<x$.

LEMMA 4 (COURTESY OF M. RUBIN). The homogeneous chain $\Omega$ is doubly homogeneous if and only if $\neg \rho$, where $\rho$ is the following group-theoretic statement: $\exists x, y, z$ $\in A(\Omega)$ such that $x \neq e, y \neq e, z \neq e$, and every conjugate of $z$ commutes with either $x$ or $y$.

Proof of Lemma 4. Using the fact that a $0-2$-transitive $A(\Omega)$ is also 0 - $n$-transitive for every positive integer $n$, it can be shown that $\rho$ fails when $\Omega$ is doubly homogeneous. If $\Omega$ is not doubly homogeneous, there are two cases. Either $A(\Omega)$ is an Ohkuma group ( $\Omega$ is an Ohkuma segment), in which case $A(\Omega)$ is isomorphic to a subgroup of the real numbers, is therefore abelian, and so $\rho$ holds; or $A(\Omega)$ is not 
0 -primitive, and there is a proper congruence on $\Omega$ with convex classes. In this case, any nontrivial $x, y, z$ whose supports lie in different classes of that congruence show that $\rho$ holds.

Without loss of generality, the l-group language contains only $<$ in addition to the group language. Given a statement $\psi$ in the $l$-group language, we replace each occurrence of $x \leqslant y$ by $\phi_{p}(x, y)$, and call the result $\psi_{p}$. Form $\psi^{\prime}=\exists p\left(\psi_{p}\right.$ and $\exists f$ $\left.\exists g \forall u\left(\left[f,\left(u^{-1} p u\right) g\left(u^{-1} p u\right)^{-1}\right]=e\right)\right)$. Then $\psi^{\prime}$ holds in the 0-2-transitive group $A(\Omega)$ if and only if $\psi$ holds in the l-group $A(\Omega)$ or the $l$-group $A\left(\Omega^{*}\right)$ where $\Omega^{*}$ is the chain dual to $\Omega$.

\section{REFERENCES}

1. A. M. W. Glass, Ordered permutation groups, Bowling Green State University, 1976.

2. W. C. Holland, The lattice-ordered group of automorphisms of an ordered set, Michigan Math. J. 10 (1963), 399-403.

3. __ Transitive ordered permutation groups, Math. Z. 87 (1965), 420-433.

4. __ Trying to recognize the real line, Ordered Groups, Lecture Notes in Pure and Appl. Math., Dekker, New York, 1980.

5. M. Jambu-Giraudet, Théorie des modèles des groups d'automorphismes d'ensembles totalment ordonnés, Thèse $3^{\text {me }}$ cycle, Univ. Paris VII, 1979.

6. S. H. McCleary, Groups of homeomorphisms with manageable automorphism groups, Comm. Algebra 6 (1978), 497-528.

7. R. McKenzie, On elementary types of symmetric groups, Algebra Universalis 1 (1971), 13-20.

8. A. G. Pinus, On elementary defineability of symmetric groups, Algebra Universalis 3 (1973), 59-66.

9. S. Shelah, First order theory of permutation groups, Israel J. Math. 14 (1973), 149-162; 15 (1973), 437-441.

Department of Mathematics, Ben-Gurion University of the Negev, Beer Sheva, IsRael

Department of Mathematics, Bowling Green State University, Bowling Green, Ohio 43403 (Current address of W. C. Holland)

Current address (Yuri Gurevich): Institute for Advanced Studies, The Hebrew University, Jerusalem, Israel 